CONCISE REPORT

\title{
Smoking habits in patients diagnosed with ANCA associated small vessel vasculitis
}

\author{
M Haubitz, A Woywodt, K de Groot, H Haller, U Goebel
}

Ann Rheum Dis 2005;64:1500-1502. doi: 10.1136/ard.2004.033191

\begin{abstract}
Objectives: To study the prevalence of smoking at onset of symptoms in patients with small vessel vasculitis associated with antineutrophil cytoplasmic antibodies (ANCA).

Methods: A retrospective study, in 197 patients with ANCA associated vasculitis, of the history of cigarette smoking at onset of symptoms. Prevalence of smoking in patients with ANCA associated vasculitis was compared with age-specific values for the general population in Germany.

Results: 27 (14\%) patients were smokers at the time of first disease manifestation $(p<0.001$, compared with the entire population); 54 (27\%) had smoked previously with 1-110 pack-years (median 18) but had stopped $\geqslant 2$ years before onset of vasculitis; 116 (59\%) patients were lifelong nonsmokers. At onset of symptoms, active smokers were younger (median age 42 years) than patients with vasculitis (median 54 years, $p<0.01$, Mann-Whitney $U$ test) with a lower percentage of women $(15 \%, p<0.005$, Fisher's exact test) than in the entire group (47\%). Smokers, non-smokers, or exsmokers did not differ in organ manifestation, mortality, and development of end stage renal disease and relapse rate.

Conclusions: The proportion of active smokers in the group of patients with ANCA associated vasculitis is significantly lower than in the entire population. Cigarette smoking may be associated with a reduced risk of ANCA associated vasculitis.
\end{abstract}

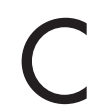
igarette smoking is a major health hazard, leading to cardiovascular and neoplastic disease. However, cigarette smoking confers protection against a variety of immune mediated disorders. A disease of non-smokers or those who have recently stopped smoking, ${ }^{1}$ ulcerative colitis serves as a paradigm. Accordingly, transdermal nicotine has been proposed and direct effects of nicotine on immune mechanisms $^{2}$ have been postulated. Similarly, cigarette smoking has been reported to confer protection against coeliac disease. ${ }^{3}$ In contrast, data on the role of smoking in vasculitis are scant ${ }^{4}$ and limited to the subset of rheumatoid vasculitis. We were interested in the prevalence of smoking in small vessel vasculitis associated with antineutrophil cytoplasmic antibodies (ANCA). Here, we report smoking habits at the onset of symptoms in a large cohort of such patients at two major German referral centres.

\section{PATIENTS AND METHODS}

The study was jointly undertaken by two referral centres for ANCA associated vasculitis in Germany, which serve the north of Germany and the Berlin/Brandenburg area, respectively. We attempted to interview all outpatients with ANCA associated vasculitis treated at Hannover Medical School and Franz-Volhard Clinic. Wegener's granulomatosis was diagnosed according to the American College of Rheumatology criteria and the Chapel Hill Consensus Conference definition, microscopic polyangiitis according to the Chapel Hill Consensus Conference definition. Patients with ChurgStrauss syndrome were excluded, because obstructive pulmonary disease may have specific influence on smoking habits.

Patients were interviewed during their routine appointments or by telephone, and informed consent was obtained. Current and former smoking habits, start and cessation of smoking, and pack-years were documented. For this study, a smoker was defined as someone who smoked cigarettes daily (with a minimum of one cigarette a day) at the onset of symptoms or within the preceding 2 years. Smoking of cigars, cigarillos, and pipes was also inquired about. Clinical data were retrieved from files. The prevalence of smoking was compared with data obtained from the 1995 microcensus. These data derive from a federal randomised survey of 390000 households with 830000 people ( 15 years or older), equalling $1 \%$ of the German population. The proportion of smokers among patients with vasculitis was compared with that within the general population with binomial testing. Comparisons of age were done with the Mann-Whitney U test. Binary variables were compared using Fisher's exact test.

\section{RESULTS}

One hundred and ninety seven patients were identified and follow up time was between 2 and 376 months (median 85 months). One hundred and thirty two patients had Wegener's granulomatosis and 65 had microscopic polyangiitis. Their age was 15-82 years (median 54 years) and 92 $(47 \%)$ were female. Forty seven patients were undergoing dialysis and relapse rate was 1.2 relapse episodes per patient. The disease manifestation was renal in $166(84 \%)$ patients, pulmonary in $139(71 \%)$, and ear/nose/throat in $132(67 \%)$. Forty seven (24\%) had cutaneous vasculitis, 17 (9\%) had vasculitis of the central nervous system, $55(28 \%)$ had peripheral nerve disease, 59 (30\%) had eye disease, and 133 (68\%) had arthralgia; 29 (15\%) had an organ disease other than those mentioned above.

Only $27(14 \%)$ patients were smokers at the time of first disease manifestation. Fifty four $(27 \%)$ had smoked earlier in life with 1-110 pack-years (median 18) but had stopped smoking at least 2 years before manifestation of the vasculitis. Daily smoking of cigars, cigarillos, and pipes did not occur. One hundred and sixteen (59\%) patients were lifelong non-smokers. Figure 1 shows the age distribution and smoking status. The proportion of smokers among patients with vasculitis was significantly lower than in the general German population $(24.3 \%$ smokers, $p<0.001$, binomial testing). This difference was seen for both men and women separately $(\mathrm{p}<0.05$ in men and $\mathrm{p}<0.001$ in women).

At the onset of disease, active smokers with vasculitis were younger (median age 42 years) than all patients with 


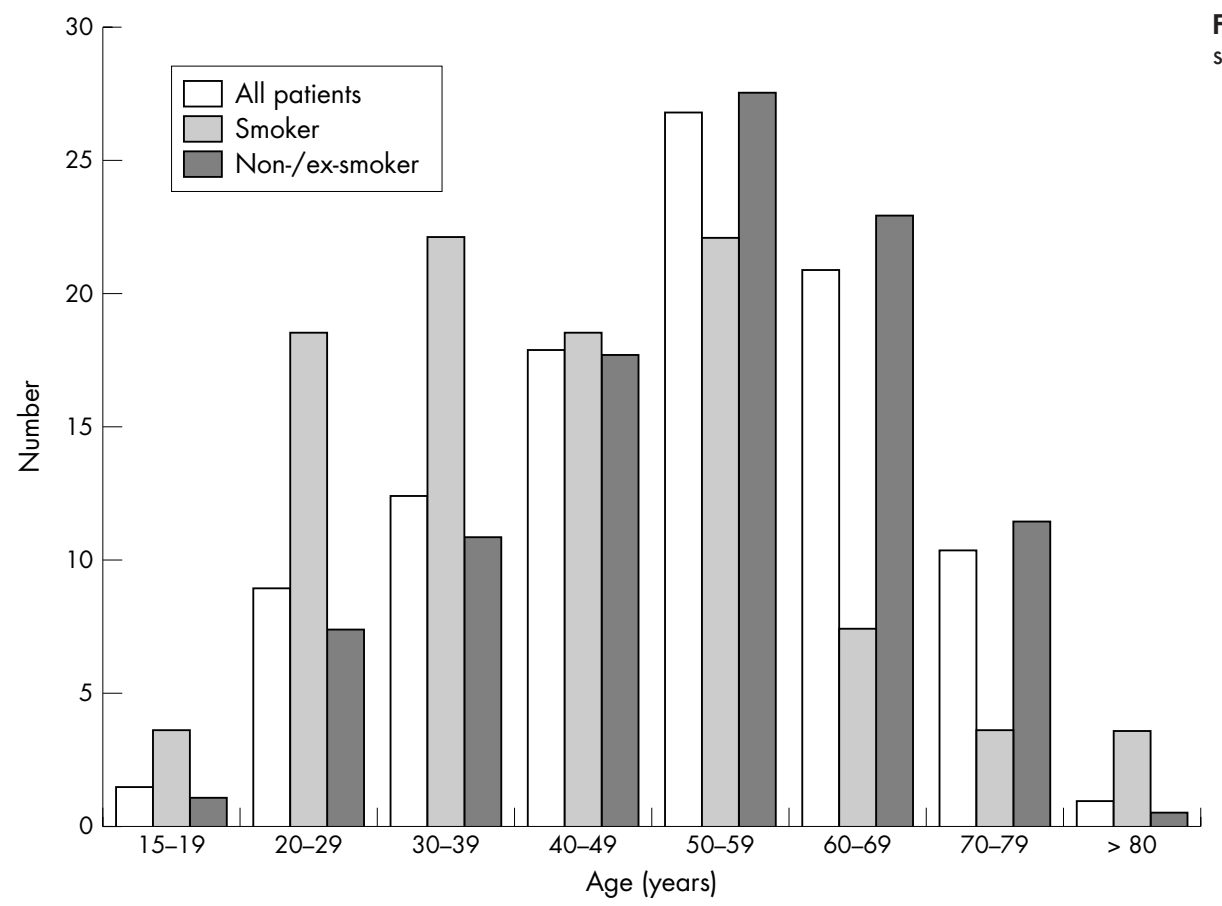

Figure 1 Age distribution and smoking status.

vasculitis (median 54 years, $\mathrm{p}<0.01$ ). Among smokers with vasculitis there was a lower percentage of women ( $15 \%(5 / 27$ patients), $p<0.005)$ than in the entire group $(47 \%(92 / 197)$. Smokers, non-smokers, or ex-smokers did not differ in organ manifestation, mortality, and development of end stage renal disease and relapse rate.

\section{DISCUSSION}

Patients who smoke are generally discouraged from doing so on the basis of a robust association between cigarette smoking, cardiovascular disease, and cancer. On the other hand, however, smoking may ameliorate specific diseases. In this regard, autoimmune disorders are of particular interest. Studies into such associations may be of interest for clinicians, in that smoking status may influence the likelihood of a particular diagnosis. In addition, such observations may trigger new treatment options. Accordingly, transdermal nicotine treatment has been advocated in ulcerative colitis. ${ }^{5}$ The efficacy of such treatment supports the assumption that nicotine itself mediates the beneficial effect of smoking in ulcerative colitis. More and more effects of nicotine on the immune system have recently emerged, not least a direct effect on T cell receptor mediated signalling. ${ }^{6}$ The importance of these mechanisms, however, remains controversial. More recently, the haem oxygenase system has emerged as a new immune mechanism, ${ }^{7}$ with carbon monoxide as its chief product.

We describe, for the first time, a significantly lower prevalence of smoking in patients with ANCA associated small vessel vasculitis than in the general population. This finding is quite surprising, given that cigarette smoking causes direct endothelial damage. ${ }^{8}$ Therefore, one would assume that cigarette smoking propagates and aggravates any subtype of vasculitis. Accordingly, a higher prevalence of smoking has been reported in patients with rheumatoid vasculitis. ${ }^{4}$ Another small study in 28 patients described a higher prevalence of smoking in patients with rapidly progressive glomerulonephritis due to ANCA associated small vessel vasculitis. ${ }^{9}$ Finally, our report is in contrast with another study into environmental factors that failed to show any association between smoking status and ANCA associated vasculitis. ${ }^{10}$ In contrast with our work, however, that study was not intended to study smoking habits but focused on occupation and exposure to chemicals.

Our study has several limitations. Firstly, our patients were recruited from two divisions of nephrology. Although both departments see patients with and without renal disease, a bias towards renal and more severe disease cannot be excluded. Secondly, the study was retrospective and patients' recall may have been inaccurate. Moreover, it is conceivable that patients quitted smoking while feeling generally unwell before the onset of more specific symptoms. Although caused by vasculitis, such malaise may later be ascribed to other factors. To avoid such bias, patients who had stopped smoking within 2 years before the onset of symptoms were also defined as smokers. Finally, smoking differs markedly within the general population. A higher prevalence of smoking has, for example, been reported in people with low social status and after divorce. Higher socioeconomic groups with a low prevalence of smoking may also be more likely to present to tertiary care hospitals. Regarding these factors, we do not believe that the patients with vasculitis differed markedly from the general population.

Another influence is that of region-for example, in Germany smoking is more prevalent in the north. According to these data, an even higher prevalence of smoking would have to be expected in our patients, thus adding to the significance of our observation. Unfortunately, our patients came from several areas-namely, Lower Saxony, Hessia, North-Rhine-Westphalia, and SachsenAnhalt for the Hannover unit and Berlin as well as Brandenburg for the Berlin unit. A comparison of smoking prevalence in our patients with specific data from just one of these regions would therefore be inappropriate.

We report, for the first time, a significantly lower prevalence of smoking at the onset of vasculitic symptoms as compared with the general population in Germany. It is tempting to speculate about the reasons for this. Conceivably, some unknown environmental factor is involved in the pathogenesis of vasculitis and associated with non-smoking or stopping smoking. Various environmental factors have 
previously been studied in ANCA associated vasculitis. ${ }^{10}$ Most notably, associations with silica exposure, farming, and solvents have been reported. Among those, the association with exposure to silica appears to be most robust and confirmed by several studies. ${ }^{11}$ Unfortunately, data on the prevalence of smoking by occupation in Germany are not available. When gauged by data from the United States, however, mining is associated with a higher prevalence of smoking, whereas farming is associated with a lower prevalence. ${ }^{12}$ It must be emphasised, however, that such figures may differ markedly between countries. In summary, a factor independent of silica exposure or farming may be responsible.

Finally, one may speculate as to pathogenetic mechanisms, because nicotine and the carbon monoxide system may influence immune events during the pathogenesis of vasculitis. The importance of $\mathrm{T}$ cells in vasculitis has been emphasised $^{13}$ and nicotine has been shown to impair $\mathrm{T}$ cell signalling. ${ }^{14}$ Specifically, it has been reported that nicotine impairs Fas/Fas ligand signalling in lymphocytes. ${ }^{15}$ Carbon monoxide has been shown to inhibit apoptosis of vascular smooth muscle cells, regulate vascular tone, and inhibit thrombosis. The importance of such events in transplantation $^{16}$ and in the kidney has been emphasised elsewhere. ${ }^{17}$ Whether inhaled carbon monoxide may have equally beneficial effects remains unclear. ${ }^{18}$ It is tempting to speculate that inhaled carbon monoxide from cigarette smoke may inhibit immune mechanisms involved in the development of vasculitis, more so because early events in ANCA associated vasculitis often occur in upper airway tissue, where carbon monoxide would be expected to act.

In summary, pathogenetic mechanisms by which smoking might protect against small vessel vasculitis may involve nicotine, carbon monoxide, or other substances in cigarette smoke. These mechanisms clearly deserve further study, although such research is hampered by a paucity of animal models for ANCA associated small vessel vasculitis.

\footnotetext{
Authors' affiliations

M Haubitz, A Woywodt, K de Groot, H Haller, Department of

Nephrology, Hannover Medical School, Hannover, Germany

U Goebel, Department of Nephrology, Helios Kliniken, Franz-Volhard Hospital, Charité Berlin-Buch, Berlin, Germany

Competing interests: The authors declare no competing interests.
}

Correspondence to: Dr M Haubitz, Department of Nephrology, Hannover Medical School, Carl-Neuberg-Strasse-1, 30625 Hannover, Germany; Haubitz.Marion@MH-Hannover.de

Accepted 14 March 2005

\section{REFERENCES}

1 Boyko EJ, Koepsell TD, Perera DR, Inui TS. Risk of ulcerative colitis among former and current cigarette smokers. N Engl J Med 1987;316:707-10.

2 Eliakim R, Fan QX, Babyatsky MW. Chronic nicotine administration differentially alters jejunal and colonic inflammation in interleukin-10 deficient mice. Eur J Gastroenterol Hepatol 2002;14:607-14.

3 Suman S, Williams EJ, Thomas PW, Surgenor SL, Snook JA. Is the risk of adult coeliac disease causally related to cigarette exposure? Eur J Gastroenterol Hepatol 2003;15:995-1000.

4 Struthers GR, Scott DL, Delamere JP, Sheppeard H, Kitt M. Smoking and rheumatoid vasculitis. Rheumatol Int 1981;1:145-6.

5 Pullan RD, Rhodes J, Ganesh S, Mani V, Morris JS, Williams GT, et al. Transdermal nicotine for active ulcerative colitis. N Engl J Med 1994:330:81 1-15.

6 Sopori M. Effects of cigarette smoke on the immune system. Nat Rev Immunol 2002;2:372-7

7 Morse D, Choi AM. Heme oxygenase-1: the "emerging molecule" has arrived. Am J Respir Cell Mol Biol 2002;27:8-16.

8 Blann AD, McCollum CN. Adverse influence of cigarette smoking on the endothelium. Thromb Haemost 1993;70:707-11.

9 Sessa A, Meroni M, Battini G, Vaccari M, Giordano F, Torri Tarelli L. Cigarette smoking and pauci-immune extracapillary glomerulonephritis with ANCAassociated idiopathic systemic vasculitis. Contrib Nephrol 2000;130:103-8.

10 Lane SE, Watts RA, Bentham G, Innes NJ, Scott DG. Are environmental factors important in primary systemic vasculitis? A case-control study. Arthritis Rheum 2003:48:814-23

11 Hogan SL, Satterly KK, Dooley MA, Nachman PH, Jennette JC, Falk RJ. Silica exposure in anti-neutrophil cytoplasmic autoantibody-associated glomerulonephritis and lupus nephritis. J Am Soc Nephrol 2001;12:134-42.

12 NIOSH NIfOSaH. Occupational Respiratory Disease Surveillance: Smoking Prevalence by Occupation and Industry, 2000. http://www.cdc.gov/niosh/ topics/surveillance/ords/NationalStatistics/WoRLDHighlightsSmoking.html, accessed 4 July, 2005

13 Clayton AR, Savage CO. What you should know about PR3-ANCA. Evidence for the role of T cells in the pathogenesis of systemic vasculitis. Arthritis Res 2000;2:260-2.

14 Sopori ML, Kozak W, Savage SM, Geng Y, Soszynski D, Kluger MJ, et al. Effect of nicotine on the immune system: possible regulation of immune responses by central and peripheral mechanisms. Psychoneuroendocrinology 1998;23:189-204.

15 Suzuki N, Wakisaka S, Takeba Y, Mihara S, Sakane T. Effects of cigarette smoking on Fas/Fas ligand expression of human lymphocytes. Cell Immunol 1999; 192:48-53.

16 Katori M, Busuttil RW, Kupiec-Weglinski JW. Heme oxygenase-1 system in organ transplantation. Transplantation 2002;74:905-12.

17 Hill-Kapturczak N, Chang SH, Agarwal A. Heme oxygenase and the kidney DNA Cell Biol 2002;21:307-21.

18 Thiemermann C. Inhaled CO: deadly gas or novel therapeutic? Nat Med $2001 ; 7: 534-5$. 